\title{
On the overlap between bilingual language control and domain- general executive control
}

\author{
Francesca M. Branzi ${ }^{1,2,3}$, Marco Calabria ${ }^{3}$, Maria Lucrezia Boscarino ${ }^{3}$, Albert \\ $\operatorname{Costa}^{3,4}$ \\ ${ }^{1}$ Neuroscience and Aphasia Research Unit, School of Psychological Sciences, \\ University of Manchester, UK \\ 2 Basque Center on Cognition, Brain and Language (BCBL), San Sebastian, Spain \\ ${ }^{3}$ Center for Brain and Cognition (CBC), Universitat Pompeu Fabra, Barcelona, Spain \\ ${ }^{4}$ Institució Catalana de Recerca i Estudis Avançats (ICREA), Barcelona, Spain
}

\section{Address for correspondence:}

Francesca M. Branzi

Room 3.19, Neuroscience and Aphasia Research Unit (NARU)

Zochonis Building, University of Manchester,

Brunswick Street, Manchester, M13 9PL

Tf. +440345600043

email francesca.branzi@gmail.com 


\begin{abstract}
We explored the overlap between bilingual language control (bLC) and domain-general executive control (EC) by focusing on inhibitory control processes. We tested 62 bilinguals in linguistic and non-linguistic switching tasks for two types of costs, such as the n- 1 shift cost and the n- 2 repetition cost. In order to explore the involvement of inhibitory control in bLC and EC, we assessed the pattern of switch costs in the two tasks and then we correlated them between tasks. Results showed reduced $n-2$ repetition costs as compared to $n-1$ shift costs in the linguistic task only, suggesting that small amount of inhibition were deployed when switching between languages. Importantly, neither the $n-1$ shift costs nor the $n-2$ repetition costs were correlated between tasks. These results, supported by additional evidence from the ex-Gaussian analysis, suggest that inhibitory control is differently involved in bLC and in EC.
\end{abstract}

Keywords: Bilingual language control, domain-general executive control, inhibitory control, task-switching. 


\section{Introduction}

The issue of how bilingual speakers manage to restrict lexicalization to one of their languages, while preventing massive interference from their other language, has prompted a great amount of research in the last decades (e.g., Costa \& Santesteban, 2004; Costa, Santesteban \& Ivanova, 2006; Jackson, Swainson, Cunnington, \& Jackson, 2001; Christoffels, Firk, \& Schiller, 2007; Misra, Guo, Bobb, \& Kroll, 2012; Branzi, Martin, Abutalebi \& Costa, 2014; Branzi, Della Rosa, Canini, Costa \& Abutalebi, 2015; for a review see Baus, Branzi \& Costa, 2015). As a result of this research, there is agreement in assuming that the bilingual language control (bLC) system makes use of various processes of the domain-general executive control (EC) system (e.g., Abutalebi \& Green, 2007). However, the precise nature of the overlap between bLC and EC processes is still an open issue. The aim of this article is to provide new evidence regarding the relationship between these two cognitive systems.

Recent research on the overlap between bLC and EC has focussed on different strategies. One of them is to correlate participants' behaviour in comparable tasks that either involves bLC or domain-general EC processes (e.g., Calabria, Hernández, Branzi, \& Costa, 2012; Weissberger, Wierenga, Bondi, \& Gollan, 2012; Prior \& Gollan, 2013; Calabria, Branzi, Marne, Hernández, \& Costa, 2015; Cattaneo, Calabria, Marne, Gironell, Abutalebi, \& Costa 2015; Babcock \& Vallesi, 2015). The argument made is that to the extent these tasks tap into comparable control processes, there should be a correlation between the effects measured in them. For example, Calabria et al. (2012; 2015) tested bilinguals of different ages in both linguistic and nonlinguistic switching tasks. In the linguistic task, participants were required to 
name some pictures in Catalan and some other pictures in Spanish according to a cue (i.e., Catalan and Spanish flag). In the non-linguistic task, instead, participants were required to classify pictures according to a non-linguistic classification rule (i.e., classify pictures by their color and by their shape). The cost of switching between languages or tasks was calculated by subtracting reaction times (RTs) of "repeat" trials (AA task sequences) from those of "switch trials" (BA task sequences). This cost, the so called "n-1 shift cost", is considered a measure of the efficiency of bLC and EC functioning (see Kiesel, Steinhauser, Wendt, Falkenstein, Jost, \& Philipp, 2010).

The current evidence indicates there is no correlation between linguistic and non-linguistic n-1 shift costs (Calabria et al., 2012; 2015; Prior \& Gollan, 2013; Cattaneo et al., 2015), hereby suggesting a lack of overlap between bLC and EC, at least for those cognitive mechanisms measured through the n-1 shift cost (see also Weissberger et al., 2012). In accord with these findings, Tse \& Altarriba (2014) tested a group of Cantonese-English bilingual children and revealed a lack of association between measures of language proficiency and ex-Gaussian parameters in a non-linguistic Simon switching task.

However, other behavioural studies have provided evidence of a link between bilingual language processing and EC (e.g., Festman, RodriguezFornells, \& Münte, 2010; Festman, 2012, Prior \& Gollan, 2011; Soveri, Rodriguez-Fornells, \& Laine, 2011; Hartanto, \& Yang, 2016). For instance, Soveri et al. (2011) revealed that the frequency rate with which bilinguals switched between languages on a daily basis predicted the magnitude of mixing costs in error rates in a set-shifting task. In addition to this, Goral, Campanelli and Spiro (2015) recently revealed that language use and language proficiency 
affect the performance in the Simon task in older bilinguals. Besides, it has been also demonstrated a relationship between intrusion errors in a single-language conversational context and cognitive measures of executive functioning (Festman, 2012; Gollan et al., 2011) and between measures of language control and the control of nonverbal interference (e.g., Prior \& Gollan, 2011; Linck, Schwieter, Sunderman, 2012; de Bruin, Roelofs, Dijkstra, \& FitzPatrick, 2014). Prior and Gollan (2011) showed that Mandarin-English bilinguals with higher fluency scores in Mandarin incurred smaller switch costs in a non-linguistic switching task. Note, however, that this result was not replicated in another group of bilinguals (Spanish-English bilinguals) tested in the same study.

Another indication of a link between bilingual language use and EC processing can be found in those studies that compared monolinguals and bilinguals performing EC tasks (e.g., Bialystok \& Martin, 2004; Bialystok \& Viswanathan, 2009; Prior \& MacWhinney, 2010; Tao, Marzecová, Taft, Asanowicz, \& Wodniecka, 2011; Costa, Hernández \& Sebastián-Gallés, 2008; Costa, Hernández, Costa-Faidella, \& Sebastián-Gallés, 2009; Wiseheart, Viswanathan, \& Bialystok, 2016). In fact, some of these studies revealed that the continuous use of two languages affect the cognitive processes related to domain-general EC, such as those put at play during non-linguistic switching tasks (e.g., Prior \& MacWhinney, 2010; Prior \& Gollan, 2011; Prior, 2012; Wiseheart, et al., 2016), thereby suggesting a certain functional overlap between those processes involved in linguistic and non-linguistic domains of cognitive control. Despite these findings have motivated recent research to focus on the extent of this overlap (e.g., Calabria et al., 2012; Weissberger et al., 2012; Cattaneo et al., 2015; Coderre, Smith, van Heuven, \& Horwitz, 2015; 
Weissberger, Gollan, Bondi, Clark, \& Wierenga, 2015; De Baene, Duyck, Brass, \& Carreiras, 2015), other recent findings have strongly undermined the basic assumption of the existence of an overlap between bLC and EC (e.g., Paap et al., 2013; Hernandez et al., 2013; Paap \& Sawi, 2014; Duñabeitia, Hernández, Antón, Macizo, Estévez, Fuentes \& Carreiras, 2014; Kousaie, Sheppard, Lemieux, Monetta, \& Taler 2014; Antón, Duñabeitia, Estévez, Hernández, Castillo, Fuentes, Davidson, \& Carreiras, 2014; von Bastian, Souza, Gade, 2015).

Hence, at present, the available evidence is contradictory regarding the existence of a substantial overlap between bLC and EC processes, particularly as to when such overlap is measured by correlating switch costs between tasks (Calabria et al., 2012; 2015; Prior \& Gollan, 2013).

Informative as this lack of correlation might be, indeed, it might not tackle specifically those control processes that are supposed to be influenced by bilingualism, such as inhibitory control processes (e.g., Green, 1998). In fact, despite the $\mathrm{n}-1$ shift cost measures inhibitory control processes, it reflects also the efficiency of other EC mechanisms involved in switching between tasks (e.g., task-set activation mechanisms; see Kiesel et al., 2010). Hence, the lack of correlation between linguistic and non-linguistic tasks might be due to the variability added by these other processes measured through the $\mathrm{n}-1$ shift cost.

One of the aims of this study is to assess this issue by measuring a cognitive cost that is supposed to tackle specifically inhibitory control processes and that thus may reveal a correlation between the two tasks. This cost is the "n-2 repetition cost" (e.g., Mayr \& Keele, 2000; Philipp, Gade, \& Koch, 2007; see also Koch, Gade, Schuch, \& Philipp, 2010) and it refers to the slower RTs observed when participants have to switch into a recently performed task (in an n-2 
trial) as compared to when they have to switch into a not-recently performed task. To give an example, let's consider a switching experiment in which participants have to switch between three different tasks (sort pictures by color, size and shape). This task affords the calculation of the cost of switching into a recently performed task (ABA-classify by color, classify by size, classify by color), and that of switching into a not recently performed task (CBAclassify by shape, classify by size, classify by color). As it happens, RTs from the former type of trials are slower than those of the later, the so-called n-2 repetition cost (e.g., Mayr \& Keele, 2000; Philipp et al., 2007). As hinted above, the magnitude of this cost is argued to be a signature of the amount of inhibition applied to the repeated task. In other words, the inhibition applied to task A when performing task B would determine an increase of RTs when performing again task A, because of the need to overcome previous inhibition (e.g., Arbuthnott \& Frank, 2000; Mayr \& Keele, 2000).

The overlap between bLC and EC has not yet been assessed by measuring this index of inhibitory control (but see Babcock \& Vallesi, 2015 for some evidence with simultaneous interpreters). Hence, with this study we aim to fill this gap, by exploring not only between-tasks correlations of the n- 1 shift cost (Calabria et al., 2012, 2015), but also those of the n-2 repetition cost. Importantly, providing an answer to this question is a timely issue given the current debate on the existence of bilingualism advantages in inhibitory control (e.g., Paap, Johnson, \& Sawi, 2015). In that, the finding of an association between linguistic and non-linguistic n-2 repetition costs would contrast with the recent views that suggest the bilingual advantage in inhibitory control is not a reliable phenomenon (Paap et al., 2013; Paap et al., 2015). 
Under the assumption that the $\mathrm{n}-2$ repetition cost is a reliable index of inhibitory control ${ }^{1}$, we hypothesize that participants' variability in their inhibitory abilities should be revealed by differences in the magnitude of the n2 repetition cost. If bLC makes use of the same inhibitory processing as the domain-general EC system, then participants' variability in their inhibitory abilities should be similarly present in linguistic and in non-linguistic tasks. In other words, the n-2 repetition cost should correlate between bLC and EC tasks.

In this study, we use an experimental design that affords exploring both the n- 1 shift cost and the $n-2$ repetition cost simultaneously. We do so, because the assessment of the patterns of switch costs (n-1 shift costs and n-2 repetition costs) may be informative in respect of the involvement of inhibitory control in the two tasks. Let us explain in more detail the pattern of switch costs we are referring to.

As previously hinted, both the n-1 shift cost and the $n-2$ repetition cost are cognitive indexes that capture inhibitory control in the task. However, our hypothesis is that in doing so they may show opposing effects. That is, based on the assumption that inhibition measured as $n-2$ repetition costs contribute to n-1 shift costs (Mayr \& Keele, 2000), the stronger the inhibition applied on the n-1 task, the smaller the $n-1$ shift cost and the larger the $n-2$ repetition cost should be.

\footnotetext{
1 The $n-2$ repetition cost is considered a robust and reliable index of inhibitory control. In fact, to our knowledge, besides very few exceptions (e.g., Guo, Ma, \& Liu, 2013), all the studies that measured this index reported significant n-2 repetition costs (see, e.g., Arbuthnott \& Frank, 2000; Lien \& Ruthruff, 2008; Mayr \& Keele, 2000; Philipp, Jolicoeur, Falkenstein, \& Koch, 2007; Schuch \& Koch, 2003; Moritz, Hübner, \& Kluwe, 2004; Whitmer \& Banich, 2007; Declerck, et al., 2015; Grange \& Juvina 2015; Babcock \& Vallesi, 2015; Scheil, 2016: Regev \& Meiran, 2016). Importantly, to date the evidence indicating that the $\mathrm{n}-2$ repetition cost reflects inhibitory control is robust against non-inhibitory explanations (see Koch et al., 2010; Mayr, 2007).
} 
Therefore, if different amount of inhibitory control are deployed when switching between non-linguistic and linguistic tasks, we may observe different patterns of switch costs in the two tasks. This prediction has not been tested so far. With the present study we aim to shed light on this issue by measuring the two costs simultaneously in linguistic and non-linguistic switching tasks.

\subsection{The present study}

In the present study, we test 62 early and high-proficient bilinguals in linguistic and non-linguistic switching tasks to explore the cognitive overlap between bLC and EC systems. Specifically, we assess whether bLC and EC tasks engage the very same inhibitory control mechanisms by conducting different comparisons both within and between tasks.

In order to reveal the involvement of inhibitory control in the two tasks we compare the magnitudes of the $\mathrm{n}-1$ shift cost and the $\mathrm{n}-2$ repetition cost within each task.

First, we hypothesize that if during bLC and EC the same amount of inhibition is applied on the n-1 task, the difference between the magnitudes of the two costs will be similar in the two tasks, regardless of the overall magnitude of these effects across tasks. Hence, we explore how much the magnitude of the $n-2$ repetition cost departs from that of the $n-1$ shift cost in linguistic and in non-linguistic switching tasks as separately.

We hypothesize that if high-proficient bilinguals do not control their languages through inhibitory control (see Costa et al., 2004; 2006), in the linguistic task we should not observe any n-2 repetition cost. This is because, if 
the amount of inhibition applied on the just executed language corresponds to zero, the expected magnitude of the $n-2$ repetition cost would be a negative value (repetition priming facilitation). Moreover, we should find the magnitude of the $n-2$ repetition cost to be smaller than that of the $n-1$ shift cost. This is because the lack of inhibition on the $n-1$ task should eliminate the $n-2$ repetition cost and at the same time enlarge the $\mathrm{n}-1$ shift cost.

Importantly, if these effects are specific to the linguistic domain (see Costa et al., 2004; 2006), we should observe a different pattern of switch costs in the non-linguistic switching task. For instance, if inhibitory control is strongly applied on the just executed task (n-1 task), we may observe in the same participants larger $n-2$ repetition costs than $n-1$ shift costs.

Second, we hypothesize that if both $n-1$ shift costs and n-2 repetition costs origin from the same mechanism (e.g., inhibition), we should observe a negative correlation between the magnitude of $n-1$ shift costs and that of the n2 repetition costs, irrespective of the task.

Finally, as in previous studies (e.g., Calabria et al., 2012; 2015), we hypothesize that between-tasks correlations of the two switch costs would be informative about the degree of the overlap between the bLC and EC systems. Specifically, we hypothesize that if inhibitory control is the specific mechanism shared between the two systems, the $n-2$ repetition cost should be correlated between tasks. 


\section{Materials and Methods}

\subsection{Participants}

Sixty-two early and high-proficient Catalan/Spanish bilinguals (46 females, mean age $=21$ years \pm 2 ) took part in the study. All participants were righthanded and had normal or corrected-to-normal vision. Languages' information and self-assessed proficiency for bilinguals is reported in Table 1.

Please insert Table 1 around here

\subsection{Materials and procedure}

In the present experiment all participants were presented with a linguistic and a non-linguistic switching task, administered in different days (order of presentation was counterbalanced; the interval between the first and the second experiment was approximately of one week). After having filled a language proficiency questionnaire, each participant was tested individually in a soundproof room. At the beginning participants received written and oral experimental instructions and then they took part in the experiment through a single session of approximately 45 minutes. Instructions emphasized speed and accuracy. Participants were informed about the tasks and the responses, but we did not mention the presence or absence of repetition trials. Before being tested in both experiments, all participants were trained with a practice session. 


\subsubsection{Linguistic switching task}

Before the experiment started, participants were familiarized with the pictures used in the experiment, in order to verify that they could produce the wanted names for each one of the pictures.

In the linguistic switching task, participants were presented with eight pictures of concrete objects (Snodgrass \& Vanderwant, 1980). These pictures were all non-cognate words, that is, words with the same meaning but distinct phonology in three naming languages (i.e., Catalan, Spanish and English): carrot [zanahoria (Spanish), pastanaga (Catalan)]; sock [calcetín, mitjó]; cage [jaula, gàbia]; duck [pato, ánec]; butterfly [mariposa, papallona]; pillow [almohada, coixí]; apple [manzana, poma]; cheese [queso, formatge].

Participants were required to name pictures aloud by alternating between the three languages (Catalan=L1; Spanish=L2 and English=L3). Pictures were presented one at time at the centre of a white screen. The naming language was indicated by four cue-signs (flags) surrounding the picture. Responses were given verbally and were recorded through a microphone.

The experiment consisted of 6 blocks of 108 trials each. Each trial started with a blank screen followed by the presentation of the cue-signs. After $100 \mathrm{~ms}$ (CSI), the picture was presented in the middle of the screen, simultaneously with a tone. Picture and cue-signs remained on the screen until the response was given (or with a maximum delay of $7000 \mathrm{~ms}$ ). The response-stimulus interval (RSI) was maintained constant (1100 ms) as well as the cue-stimulus interval (CSI) (100 ms). At the end of each block participants could take a break, and the start of the successive block was self-paced. 
Importantly, the magnitude of the $\mathrm{n}-2$ repetition cost might be affected by the presence of repetition trials (see Philipp \& Koch, 2006). This appears to be the case when the number of $n-1$ repetition trials (CAA) is equivalent to that of the $n-2$ switch (CBA) and $n-2$ repetition (ABA) trials (see Experiment 1 in Philipp \& Koch 2006). However, if the overall number of repetition trials (i.e., CAA trials) presented in the task is notably reduced as compared to the other types of trials (i.e., CBA and ABA trials), the $n-2$ repetition cost is not eliminated (see Experiment 2 in Philipp \& Koch 2006). Hence, since we aimed to measure the two costs within the same experimental design, we decided to introduce in both tasks fewer repetitions (CAA task sequences) as compared to the other two conditions (CBA and ABA task sequences).

The ABA (or $n-2$ repetition) sequence occurred with a probability of $39 \%$, the CBA (or $n-2$ switch) sequence with a probability of $39 \%$, and the CAA sequence (or $n-1$ repetition) with a probability of $11 \%$ (note that the sum of probabilities is lower than $100 \%$ because trials following a repetition were not analyzed). In the present study, we refer to A, B, C as indicating each task (i.e., name in L1, L2 and L3) that occurred in the experiment with an equal probability.

\subsubsection{Non-linguistic switching task}

In the non-linguistic switching task participants were presented with visual stimuli and were required to switch among three perceptual classification tasks. As in a previous study (Philipp \& Koch, 2006), participants were required

to classify each stimulus for the "type" (A vs. 4), the "size" (big vs. small) and the "color" (red vs. blue) accordingly with the specific cue-signs. Stimuli were 
presented one at time at the centre of a white screen. The task was indicated by four cue-signs surrounding the stimulus. The cues were paragraph signs for the "type" task, small yellow squares for the "color" task and up-down pointing arrows for the "size" task. Responses were given manually on an external keyboard with three response keys for each hand. We adopted this response setting in order not to have response overlapping across tasks. Hence, three keys were used to respond to 'A', 'big' and 'red' and three other keys were used to respond to '4', 'small' and 'blue'. Note also that responses were labeled on the keyboard. The procedure was identical to that of the linguistic switching task, with the only difference that at the end of each block participants received a feedback ${ }^{2}$ relative to their performance, in terms of the percentage of correct responses.

\section{Data analysis and Results}

As in previous studies (e.g., Philipp \& Koch, 2006), each $n$ trial was assigned to one of the three conditions (CAA, CBA and $\mathrm{ABA}$ ) depending on the nature of the two preceding trials (the n- 1 and the n-2 trials). For example, in the CBA sequence the $n$ trial $A$ is preceded by the $n-1$ trial $B$ and by the $n-2$ trial C. In this case, the $n$ trial A would be assigned to the condition $n-2$ switch, given that the trial n-1 and the trial n-2 are both different from $A$. In the ABA sequence instead, the $n$ trial A is preceded by the n-1 trial B and by the $n-2$ trial A. In this

\footnotetext{
2 The goal of providing a feedback on the performance was that of informing participants that the experimenter was monitoring online how well they were engaged in the task. In fact, participants were told that the experimenter could visualize the feedback from a computer screen outside the experimental room. In the linguistic task it was not possible to provide an online feedback, since verbal responses could not be codified online as incorrect or correct by Presentation software. However, in the linguistic task participants were told the experimenter was listening to them in order to take note of eventual errors. So, similarly as in the in the non-linguistic task, participants were informed that their performance was monitored online by the experimenter outside the room.
} 
case, the $n$ trial A would be assigned to the condition $n-2$ repetition, given that the $\mathrm{n}-2$ trial and the $n$ trial require to performing the same task (i.e., A). In the CAA sequence the $n$ trial A would be assigned to the $n-1$ repetition condition, given it is preceded by an identical trial, that is, the n- 1 trial A.

The n-1 shift cost was calculated by subtracting the RTs of the CAA sequences from those of the CBA sequences. The $n-2$ repetition cost was calculated by subtracting RTs of the CBA sequences from those of the ABA sequences.

First, we explored RTs and the pattern of switch costs in the linguistic task and in the non-linguistic task, as separately. Hence, for each task we conducted two repeated-measure ANOVAs, one for RTs and the other for error rates, and we performed a paired t-test to compare the two "types of cost" (n-1 shift cost, $\mathrm{n}-2$ repetition cost).

Second, we further explored the overlap between bLC and EC systems by conducting the ex-Gaussian distribution analysis of RTs, for each task separately. This analysis aids to visualize behavioural trends of the dispersion that are not observable with classical measures of central tendency. Since the effects in means can be produced by shifts of RT distributions or by stretching of slow tails of RT distributions, or by a combination of both, a deeper examination of RT data may be useful. The ex-Gaussian analysis allows doing so by decomposing the overall RT distribution into two distributions: the normal and the exponential one. The normal distribution is characterized by two parameters, such as mu $(\mu)$ and sigma $(\sigma) . \mu$ is the mode of the fitted normal distribution, and $\sigma$ corresponds to the standard deviation. The exponential 
distribution, instead, corresponds to the tail of the overall RT distribution and refers to the parameter " $\tau$ ".

In attention tasks, $\mu$ and $\tau$ have been found to capture differently some cognitive indexes of EC, not only when comparing different groups of participants (e.g., Abutalebi et al., 2015), but also when investigating issues related to the overlap between bLC and EC in the same participants (Calabria et al., 2015). In this study we use this analysis to determine which parameters are specific to bLC and which are shared with the domain-general EC. Specifically, the question here is whether switch costs in the linguistic and non-linguistic tasks are similarly captured by the normal and/or by the exponential component of the RT distribution.

Third, we ran a correlation analysis between the n-1 shift cost and the n-2 repetition cost within each task, to examine whether the two costs were reflecting the same control mechanism in the linguistic and in the non-linguistic task (RTs and ex-Gaussian parameters).

Finally, we ran a correlation analysis between the two tasks in order to see whether the $n-2$ repetition cost and the $n-1$ shift cost were correlated between tasks (RTs and ex-Gaussian parameters).

In the ANOVAs' Post-hoc analyses, we consistently applied the Bonferroni correction for multiple comparisons.

In both the linguistic and the non-linguistic switching tasks, the first two trials of each block were excluded from the analyses, as well as the trials after repetitions (e.g., CAA sequences). Moreover, every error (incorrect names, verbal disfluencies or hesitations) and the two subsequent trials were 
discarded from the analyses. RTs exceeding three standard deviations above or below a participant's mean were also excluded from the analyses.

Thus, we excluded from the behavioral analyses the $18 \%(\mathrm{SD}= \pm 9)$ of the data for the linguistic switching task and the $12 \%(\mathrm{SD}= \pm 6)$ of the data for non-linguistic switching task.

\subsection{RTs and error rates}

\subsubsection{Linguistic switching task}

As previous studies (e.g., von Bastian et al., 2015) the Cronbach's alpha, an index of internal consistency of the task was calculated for the three "types of sequence" items (CAA, CBA and ABA conditions). Results indicated that the linguistic switching task had a high level of internal consistency, as determined by a Cronbach's alpha of .982 .

For the RT analysis, the variable "types of sequence" (CAA, CBA and ABA) was included in a repeated-measure ANOVA as a within-subject factor.

The main effect of "types of sequence" was significant $[F(2,122)=39.446, p$ $\left.<.001, \eta \mathrm{p}^{2}=.999\right]$, suggesting that CAA conditions (998 ms, $\mathrm{SD}=128$ ) were significantly faster than the other two [CBA (1035 ms, SD=145, p <.001, $d=.75$ ) and $\mathrm{ABA}(1047 \mathrm{~ms}, \mathrm{SD}=153, \mathrm{p}<.001, d=-1.1)]$ and that $\mathrm{ABA}$ conditions were slower than the CBA ones $(\mathrm{p}=.008, d=.38)$ (see Figure 1$)$.

In the linguistic switching task, the $\mathrm{n}-1$ shift cost (37 ms, SD=52) was larger than the $\mathrm{n}-2$ repetition cost $(13 \mathrm{~ms}, \mathrm{SD}=32)[\mathrm{t}(61)=2.728, \mathrm{p}=.008, d=.35]$ and both costs were different from zero $[\mathrm{n}-1$ shift cost: $\mathrm{t}(61)=5.589, \mathrm{p}<.001 ; \mathrm{n}-2$ repetition cost: $\mathrm{t}(61)=3.134, \mathrm{p}=.003]$ (see Figure 1$)$. 
Also for error rates analysis, the variable "types of sequence" (CAA, CBA and ABA) was included in a repeated-measure ANOVA as a within-subject factor.

The main effect of "types of sequence" was significant $[F(2,122)=5.316, p$ $\left.=.006, \eta \mathrm{p}^{2}=.83\right]$, indicating that the CBA conditions $(5.7 \%, \mathrm{SD}=4)$ were more error prone than the CAA conditions $(4.6 \%, \mathrm{SD}=4)(\mathrm{p}=.02, d=.37)$. However, the CAA conditions were not different from the ABA conditions $(5.3 \%, \mathrm{SD}=4)$ $(\mathrm{p}=.176, d=-.25)$. Similarly, also the CBA and ABA conditions elicited the same amount of errors ( $\mathrm{p}=.345, d=.2)$.

Please insert Figure 1 around here

\subsubsection{Non-linguistic switching task}

The Cronbach's alpha was also calculated for the non-linguistic switching task for the three "types of sequence" items (CAA, CBA and ABA conditions). As in the linguistic switching task, we found high level of internal consistency (Cronbach's alpha of .976).

For the RT analysis, the variable "types of sequence" (CAA, CBA and ABA) was included in a repeated-measure ANOVA as a within-subject factor.

The main effect of "types of sequence" was significant $[F(2,122)=48.470, p$ $\left.<.001, \eta \mathrm{p}^{2}>.999\right]$, suggesting that CAA conditions (984 ms, SD=183) were significantly faster than the other two [CBA (1033 ms, SD= 201, p <.001, $d=-.6$ ) 
and ABA (1074 ms, SD=199, $\mathrm{p}<.001, d=-1.19)]$ and that ABA conditions were slower than the CBA ones $(\mathrm{p}<.001, d=.77)$ (see Figure 2$)$.

In the non-linguistic switching task, the $\mathrm{n}-1$ shift cost (49 ms, $\mathrm{SD}=82$ ) and the $\mathrm{n}-2$ repetition cost $(41 \mathrm{~ms}, \mathrm{SD}=53)$ were not different $[\mathrm{t}(61)=.586, \mathrm{p}=.56$, $d=.07]$. However, they were both different from zero [n-1 shift cost: $\mathrm{t}(61)=$ 4.708, $\mathrm{p}<.001 ; \mathrm{n}-2$ repetition cost: $\mathrm{t}(61)=6.049, \mathrm{p}<.001$ ] (see Figure 2).

Also for error rates analysis, the variable "types of sequence" (CAA, CBA and ABA) was included in a repeated-measure ANOVA as a within-subject factor.

The main effect of "types of sequence" was significant $[\mathrm{F}(2,122)=8.370, \mathrm{p}$ $\left.<.001, \eta \mathrm{p}^{2}=.96\right]$, indicating that the CBA conditions $(3.4 \%, \mathrm{SD}=2)$ and the $\mathrm{ABA}$ conditions $(3.5 \%, \mathrm{SD}=3)$ were more error prone than the CAA conditions $(2.4$ $\%, \mathrm{SD}=3)(\mathrm{p}=.004, d=.45$, and $\mathrm{p}=.01, d=.36$ respectively). Moreover, the CBA and ABA conditions elicited the same amount of errors ( $p>.999, d=.06)$.

\section{Please insert Figure 2 around here}

\subsection{Ex-Gaussian analysis of RTs}

We further explored the overlap between bLC and EC by running an exGaussian analysis. To recall, the ex-Gaussian fitting decomposes the overall RT distribution into two parameters, the normal and the exponential one. The mode and standard deviation of the normal component are approximated by $\mu$ and $\sigma$ respectively, while the exponential function is approximated by $\tau$, which reflects the mean and standard deviation of the exponential component. 
In this analysis, the raw data were sorted by "types of sequence" (CAA, CBA and ABA) separately for the two tasks. The parameters of the ex-Gaussian distribution ( $\mu$ and $\tau$ ) were obtained for each participant using the quantile maximum likelihood (QML) estimation procedure in QMPE 2.18 (Cousineau, Brown, \& Heathcote, 2004). The estimation results into a value for each parameter ( $\mu$ and $\tau$ ) and for each participant per condition.

\subsubsection{Linguistic switching task}

In the linguistic switching task we performed two ANOVAs for $\mu$ and $\tau$ values separately, considering "types of sequence" (CBA, ABA and CAA) as within-subject factor.

For $\mu$, the main effect of "types of sequence" was not significant $[\mathrm{F}(2,122)=$ 2.178, $\mathrm{p}=.188, \eta \mathrm{p}^{2}=.438$ ], indicating that the CAA condition (733 ms, SD=86) had the same $\mu$ values of the CBA (738 ms, SD=98) and the ABA conditions (748 ms, $S D=89$ ). Indeed, the $n-1$ shift cost was not different from zero [ $4 \mathrm{~ms}, \mathrm{SD}=63$; $\mathrm{t}(61)=.530, \mathrm{p}=.598]$ and the $\mathrm{n}-2$ repetition cost either $(10 \mathrm{~ms}, \mathrm{SD}=46 ; \mathrm{t}(61)=$ 1.682, $\mathrm{p}=.098$ ). Paired t-test analysis revealed that the two costs were not different [t (61) = -.459, $\mathrm{p}=.648, d=-.06]$ (see Figure 3 ).

For $\tau$, the main effect of "types of sequence" was significant $[\mathrm{F}(2,122)=$ 17.97, $\left.\mathrm{p}<.001, \eta \mathrm{p}^{2}>.999\right]$, indicating that the CAA conditions (262 ms, SD=99) had smaller $\tau$ values than the CBA (297 ms, SD=102, $\mathrm{p}<.001, d=-.6$ ) and the ABA (299 ms, SD=106, $\mathrm{p}<.001, d=-.65)$ conditions $\left(\mathrm{p}_{\mathrm{s}}<.001\right)$. However, the CBA and ABA conditions did not differ ( $\mathrm{p}>.999, d=-.04)$. Accordingly, the $n-1$ shift cost (35 ms, SD=62) was different from zero [t $(61)=4.464, \mathrm{p}<.001]$. Instead, the $\mathrm{n}-2$ repetition cost $(2 \mathrm{~ms}, \mathrm{SD}=45)$ was not different from zero [ $\mathrm{t}$ 
$(61)=.406, p=.686]$. As expected, paired t-test analysis revealed that the $n-1$ shift cost was larger than the $n-2$ repetition cost $[t(61)=2.812, p=.007$, $d=.36]$.

These results suggest that the $n-1$ shift cost was captured by the exponential component ( $\tau$ values) of the RT distribution. The $\mathrm{n}-2$ repetition cost was captured by neither the exponential nor the normal component of the RT distribution. This is likely due to the fact that the linguistic n-2 repetition cost is too small to be decomposed in the two different parameters of the RT distribution.

\subsubsection{Non-linguistic switching task}

In the non-linguistic switching task we performed two ANOVAs for $\mu$ and $\tau$ values separately, considering "types of sequence" (CBA, ABA and CAA) as within-subject factor.

For $\mu$, the main effect of "types of sequence" was significant $[F(2,122)=$ 4.677, $\left.\mathrm{p}=.011, \eta \mathrm{p}^{2}=.777\right]$, indicating that the ABA condition $(667 \mathrm{~ms}, \mathrm{SD}=115)$ had larger $\mu$ values than both the CAA (644 ms, SD=119) and the CBA (647 ms, $\mathrm{SD}=107)$ conditions $(\mathrm{p}=.037, d=.33$ and $\mathrm{p}=.015, d=.38$, respectively). However, the CAA conditions had the same $\mu$ values of the CBA conditions ( $\mathrm{p}>$ .999). Indeed, the $\mathrm{n}-1$ shift cost was not different from zero [3 ms, $\mathrm{SD}=66$; $\mathrm{t}$ (61) $=.348, \mathrm{p}=.729]$. Instead, the $\mathrm{n}-2$ repetition cost was different from zero $(20$ $\mathrm{ms}, \mathrm{SD}=53 ; \mathrm{t}(61)=2.913, \mathrm{p}=.005)$. The two costs did not differ $[\mathrm{t}(61)=-$ 1.332, $\mathrm{p}=.188, d=-.17$ ] (see Figure 3).

For $\tau$, the main effect of "types of sequence" was significant $[\mathrm{F}(2,122)=$ 34.962, $\left.\mathrm{p}<.001, \eta \mathrm{p}^{2}>.999\right]$, indicating that the CAA condition $(337 \mathrm{~ms}, \mathrm{SD}=$ 
134) had smaller $\tau$ values than both the CBA (385 ms, SD $=159, \mathrm{p}<.001, d=-$ .72 ) and the ABA conditions (405 ms, $\mathrm{SD}=157, \mathrm{p}<.001, d=-1.01$ ). Further, the ABA conditions had larger $\tau$ values than the CBA conditions ( $\mathrm{p}=.012, d=.381)$. Results revealed that both the $n-1$ shift cost (48 ms, SD=71) and the $n-2$ repetition cost $(20 \mathrm{~ms}, \mathrm{SD}=52)$ were different from zero $[\mathrm{n}-1$ shift cost: $\mathrm{t}(61)=$ 5.291, $\mathrm{p}<.001 ; \mathrm{n}-2$ repetition cost: $\mathrm{t}(61)=2.987, \mathrm{p}=.004]$. Paired t-test between the two costs revealed that the n-1 shift cost was larger than the $n-2$ repetition cost $[\mathrm{t}(61)=2.133, \mathrm{p}=.037, d=.27]$.

These results suggest that in the non-linguistic switching task the $\mathrm{n}-1$ shift cost was captured by the exponential component, whereas the $n-2$ repetition cost was captured by both the normal and the exponential parameters.

\subsection{Comparisons within tasks: Correlation analysis of the switch}

\section{costs}

\subsubsection{RTs}

In order to explore whether a common control mechanism is measured by the two costs we correlated (Pearson's coefficient) the n-1 shift cost and the n- 
2 repetition cost within each task. Interestingly, we found negative correlations between the two costs, both in the linguistic $[r=-.356, p=.005]$ and in the nonlinguistic switching tasks $[\mathrm{r}=-.413, \mathrm{p}=.001]$ (see Figure 4).

\subsubsection{Ex-Gaussian parameters: $\mu$ and $\tau$}

In order to reveal whether these correlations could affect the components of the RT distribution similarly, we also explored the correlation (Pearson's coefficient) between the $n-2$ repetition cost and the $n-1$ shift cost, for $\mu$ and $\tau$ separately. In fact, it could be that the correlation between the costs in the two tasks is mainly driven by one or both of these parameters.

For $\mu$, we found negative correlations between the two costs, both in the linguistic $[\mathrm{r}=-.558, \mathrm{p}<.001]$ and in the non-linguistic switching tasks $[\mathrm{r}=-$ $.354, \mathrm{p}=.005]$.

For $\tau$, we found negative correlations between the two costs, both in the linguistic $[\mathrm{r}=-.466, \mathrm{p}<.001]$ and in the non-linguistic switching tasks $[\mathrm{r}=-$ $.368, \mathrm{p}=.003]$.

Please insert Figure 4 around here 


\subsection{Comparisons between tasks: Correlation analysis of the switch}

costs

\subsubsection{RTs}

In order to evaluate the overlap between bLC and EC systems, we ran a correlation analysis (Pearson's coefficient) between the linguistic and the nonlinguistic switching tasks, for the $n-1$ shift cost and the $n-2$ repetition cost. As in previous studies (e.g., Calabria et al., 2012; 2015), we hypothesized that if the two switch costs reflect the efficiency of the bLC and EC systems, we may expect that the magnitudes of the switch costs (linguistic and non-linguistic) vary in similar manner in the same group of participants.

Results revealed that neither the $n-1$ shift cost $(r=-.023, p=.856)$ nor the $\mathrm{n}-2$ repetition cost $(\mathrm{r}=-.051, \mathrm{p}=.695)$ were correlated between tasks.

\subsubsection{Ex-Gaussian parameters: $\mu$ and $\tau$}

In order to explore more deeply the overlap between bLC and EC systems, we ran also a correlation analysis (Pearson's coefficient) between the linguistic and the non-linguistic switching tasks for each of the two costs, for $\mu$ and $\tau$ separately.

For $\mu$, the $\mathrm{n}-1$ shift cost was not correlated between tasks $[\mathrm{r}=-.057, \mathrm{p}=$ $.658]$ and neither was the $\mathrm{n}-2$ repetition cost $[\mathrm{r}=.088, \mathrm{p}=.495]$.

For $\tau$, the $\mathrm{n}-1$ shift cost was not correlated between tasks $[\mathrm{r}=-.135, \mathrm{p}=$ $.297]$ and neither was the $\mathrm{n}-2$ repetition cost $[\mathrm{r}=.001, \mathrm{p}=.997]$. 


\section{Discussion}

In the present study we investigated the overlap between bLC and EC systems, by exploring the performance of 62 early and high-proficient bilinguals in linguistic and in non-linguistic switching tasks. The main goal of this study was to assess through different measurements whether inhibitory control was similarly involved in bLC and EC tasks.

First, we found the two costs as being correlated within both of the two tasks. This result may suggest that the $\mathrm{n}-1$ shift cost and the $\mathrm{n}-2$ repetition cost are measuring a common EC mechanism, that is, inhibitory control.

In addition to this, the magnitudes of the two costs were different in the linguistic task. Precisely, the magnitude of the n-2 repetition cost was significantly smaller than that of the $n-1$ shift cost. This result may be interpreted as reflecting that small amounts of inhibition were applied on the just used language, leading to small $n-2$ repetition costs and large $n-1$ shift costs. In the non-linguistic switching task we were expecting to observe the opposite pattern of results, that is, large n- 2 repetition costs and small n-1 shift costs. Despite we did not observe such result, nevertheless our findings suggest that a certain amount of inhibition was applied on the just used task. In fact, in the non-linguistic task the magnitude of the $n-2$ repetition cost was not smaller than that of the $n-1$ shift cost.

These results suggest that inhibitory control does not have a crucial role in language control, at least for high-proficient bilinguals. Conversely, for the same participants inhibitory control appears to have a more integral role in the non-linguistic task. 
The results from the ex-Gaussian distribution analysis support the above mentioned findings that describe the role of inhibitory control in the two tasks. In fact, when we examine the distribution of the two types of switch costs (n-1 shift cost and $n-2$ repetition cost), the exponential component $(\tau)$ captures the effects differently. That is, for the n-1 shift cost in both tasks, whereas for the n2 repetition cost only in the non-linguistic switching task.

It has been proposed that $\tau$ might reflect controlled processes related to EC, such as inhibitory control (McAuley, Yap, Christ, \& White, 2006; Shao, Roelofs, \& Meyer 2012; Spieler, Balota, \& Faust, 1996). Even though many authors refrained from interpreting the effects of this component as related to specific cognitive processes (Matzke \& Wagenmakers, 2009), the inhibitory control interpretation is in accord with our data, as it shows that $\tau$ values do not capture the $n-2$ repetition cost in the linguistic switching task, however they do so in the non-linguistic switching task.

In accord with our results, Calabria et al. (2015) reported a change in the exponential component ( $\tau$ values) in the context of the non-linguistic switching task for the effect of aging. That is, the n-1 shift cost increased in the bilingual older adults as compared to the young group and, interestingly, this relative increase was indexed by the exponential component of the distribution for switch trials ( $\tau$ values). Importantly, this age-related change was not observed in the same participants when they performed the linguistic switching task. Given that aging is also associated with a decline of the inhibitory control system (Greenwood, 2000; Rhodes, 2004; Verhaeghen \& Cerella, 2002), some researchers suggested that the exponential component captures the efficiency 
of this EC process (e.g., Penner-Wilger, Leth-Steensen, \& LeFevre, 2002; Schmiedek, Oberauer, Wilhelm, Süß, \& Wittmann, 2007; Spieler et al., 1996).

Finally, neither the $n-1$ shift cost nor the $n-2$ repetition cost was correlated across tasks, both for RTs and when data were fitted to an ex-Gaussian distribution. That is, performance in the non-linguistic task was not related to performance in the linguistic one. This result replicates previous observations regarding the n-1 shift cost (e.g., Calabria et al., 2012; Prior \& Gollan, 2013; Calabria et al., 2015), but most importantly, it shows that a reliable index of inhibitory control in task-switching (Mayr \& Keele, 2000), i.e., the n-2 repetition cost, does not correlate across tasks (see also Babcock \& Vallesi 2015).

Therefore, to the extent to which these correlations are informative on the overlap between bLC and EC systems, it seems that the mechanisms behind the two costs do not vary in the same way across bLC and EC tasks.

These results are in accord with those neuroimaging studies that failed to reveal a complete overlap between bLC and domain-general EC (e.g., Magezi, Khateb, Mouthon, Spierer, \& Annoni, 2012; Branzi et al., 2015; Blanco-Elorrieta \& Pylkkänen, 2016). For instance, in an fMRI study Branzi et al. (2015) asked to the same bilingual participants to perform both blocked linguistic and nonlinguistic switching tasks and compared the neural switch costs between the two tasks. Despite some areas such as the left prefrontal cortex, were similarly involved in the two tasks, other regions such as the anterior cingulate cortex/pre-supplementary area was differently involved in linguistic and nonlinguistic tasks. Similar findings have been provided in a recent Magnetoencephalography (MEG) study by Blanco-Elorrieta and Pylkkänen 
(2016), in which the question of the domain-generality of the mechanisms of language control have been extended also to the domain of language comprehension, leading to the similar conclusions (see also Coderre, Smith, Van Heuven, \& Horwitz, 2015).

The general pattern of the findings we provide leads to the conclusion that bLC and domain-general EC do not share the very same mechanisms and might explain why bilingual advantages in inhibitory control are difficult to replicate (see Paap \& Greenberg, 2013; von Bastian et al., 2015).

In fact, since we revealed a lack of association between linguistic and nonlinguistic measures of inhibitory control, our results support recent and robust findings that failed to encounter bilingual advantages in inhibitory control (e.g., Duñabeitia et al., 2014; von Bastian et al.. 2015; de Bruin, Bak, \& Della Sala, 2015). Hence, if we were to add the current set of results to such studies, it appears that the bilingual advantage on inhibitory control is rather elusive and very limited in the scope, if it indeed exists (Paap et al., 2015).

Be as it may, however, our findings are at odds with those studies that instead reported such advantages by employing tasks and measurements similar to ours (e.g., Prior \& MacWhinney, 2010; Prior, 2012). Particularly, in a recent study Prior (2012) found that bilinguals had larger n-2 repetition costs than monolinguals, therefore suggesting a certain overlap between inhibitory control in linguistic and non linguistic domains.

Despite these opposing outcomes might be reconciled by assuming that different types of bilinguals (i.e., those employed in our study and in that of Prior (2012)), might lead to different results, nevertheless, the lack of overlap between linguistic and non-linguistic n-2 repetition costs is problematic to be 
reconciled with the Inhibitory Control Model-IC model (Green, 1998). In fact, the basic assumption of this model is that language control is just an instantiation of motor action. As a consequence, the cognitive indexes reflecting the efficiency of language control and motor control should measure the very same mechanism. In other words, they should correlate when measured in the same participants.

Hence, to the extent to which our results undermine the assumptions of IC model, they could be seen somehow in accord with other studies that, even without addressing the issue of the domain-generality of linguistic processes, have nevertheless failed to reveal the involvement of inhibitory control during language control (e.g., Phillip et al., 2007; Runnqvist \& Costa, 2012; Runnqvist, Strijkers, Alario, \& Costa, 2012; Guo et al., 2013).

For instance, Philipp et al. (2007) did not observe the patterns of n-2 repetition costs predicted by the IC model. That is, larger n- 2 repetition costs when switching to the most dominant of the three languages (L1), as compared to the other two weaker languages (i.e., L2 and L3) and larger n-2 repetition costs when switching to L2 as compared to when switching to L3. These patterns of switch cost are expected because the IC model (Green, 1998) posits that cross-language interference is resolved by applying inhibition to the nontarget language that is proportional to its strength (level of activation); that is, the stronger the language, the more the inhibition needs to be applied. Hence, the dominant language (i.e., the L1) will be more inhibited than the weaker languages (i.e., L2 and L3). As a consequence, language re-activation will be harder for the L1 (due to the stronger inhibition) leading to larger n-2 repetition costs. Despite Phillip et al., (2007) found a larger $n-2$ repetition cost 
for the L1 as compared with the other two weaker languages, they also found a larger cost for L3 as compared to L2. A finding that is clearly at odd with the assumption of the IC model (Green, 1998).

It is important to underline that despite our results contrast with the assumptions of the IC model (Green, 1998), however, they neither are in accord with the account that proposes that high-proficient bilinguals would not resort to inhibitory control when switching between languages (see Costa et al., 2004). In fact, despite we tested early and high-proficient bilinguals we nevertheless found the $n-2$ repetition cost in the linguistic task, indicating that some inhibitory control is involved also when high-proficient bilinguals switch between languages (see also Declerck, Thoma, Koch, \& Philipp, 2015).

All in all, even if these findings might not be generalizable to other bilingual populations such as low proficient bilinguals, the results from the patterns of switch costs along with those of the correlation analyses suggest that inhibitory control does not play a crucial role in language control and that this mechanism is not shared between the bLC and EC systems, at least for what concerns early bilinguals, equally proficient in the two languages. 


\section{Conclusion}

The contribution of the present study is three-fold. First, we provide additional support to previous findings showing that control mechanisms indexed by the n-1 shift cost are differently involved in linguistic and in nonlinguistic switching tasks (e.g., Calabria et al., 2012; 2015; Weissberger et al., 2012). Second, we extended these conclusions to the $n-2$ repetition cost, a cognitive index of inhibitory control in task switching. That is, our findings indicate that inhibitory control is differently involved in bLC and in EC, at least for early and high-proficient bilinguals. Importantly, this evidence has some relevant implications for the debate on bilingual advantage in inhibitory control (see Paap et al., 2015). In fact, it suggests that advantages in inhibitory control should not be observed for high proficient bilinguals. Future research is needed to reveal whether the results relative to the $n-2$ repetition cost reflect control strategies that can be modulated by language proficiency and/or other aspects of bilingualism experience. 


\section{Acknowledgments}

This work was supported by grants from the Spanish Government (PSI201454500, PSI2008-01191, PSI2011-23033, PSI2011-23340, Consolider Ingenio 2010 CSD2007-00012), the Catalan Government (Consolidat SGR 2009-1521 and SGR 2014-1210) and by one grant from the European Research Council under the European Community's Seventh Framework (FP7/2007-2013 Cooperation grant agreement 613465-AThEME). This research received partial financial assistance as a Severo Ochoa Center of Excellence SEV-2015-0490. Francesca M. Branzi was supported by a predoctoral fellowship from the Spanish Government (FPU-20092013) and by a postdoctoral fellowship from the European Union's Horizon 2020 research and innovation programme, under the Marie Sklodowska-Curie grant agreement No 658341. Marco Calabria was supported by a postdoctoral fellowship from the Spanish Government (Ramón y Cajal Fellowship). 


\section{References}

Abutalebi, J., Guidi, L., Borsa, V., Canini, M., Della Rosa, P. A., Parris, B. A., \& Weekes, B. S. (2015). Bilingualism provides a neural reserve for aging populations. Neuropsychologia, 69, 201-210.

Abutalebi, J., \& Green, D. (2007). Bilingual language production: The neurocognition of language representation and control. Journal of Neurolinguistics, 20(3), 242-275.

Antón, E., Duñabeitia, J. A., Estévez, A., Hernández, J. A., Castillo, A., Fuentes, L. J., Davidson, D. J., \& Carreiras, M. (2014). Is there a bilingual advantage in the ANT task? Evidence from children. Frontiers in Psychology, 5, Article 398, 12 pages.

Arbuthnott, K., \& Frank, J. (2000). Executive control in set switching: Residual switch cost and task-set inhibition. Canadian Journal of Experimental Psychology/Revue canadienne de psychologie expérimentale, 54(1), 33.

Babcock, L., \& Vallesi, A. (2015). Language control is not a one-size-fits-all languages process: evidence from simultaneous interpretation students and the n-2 repetition cost. Frontiers in psychology, 6.

Baus, C., Branzi, F. M. \& Costa, A. (2015). On the mechanism and scope of language control in bilingual speech production. In John W. Schwieter. (Ed.), The Cambridge Handbook of Bilingual Processing, (pp. 508-526). Cambridge, UK: Cambridge University Press.

Branzi, F. M., Della Rosa, P. A., Canini, M., Costa, A., \& Abutalebi, J. (2015). Language Control in Bilinguals: Monitoring and Response Selection. Cerebral Cortex, bhv052. 
Branzi, F. M., Martin, C. D., Abutalebi, J., \& Costa, A. (2014). The after-effects of bilingual language production. Neuropsychologia, 52, 102-116.

Bialystok, E., \& Martin, M. M. (2004). Attention and inhibition in bilingual children: Evidence from the dimensional change card sort task. Developmental Science, 7, 325-339.

Bialystok, E., \& Viswanathan, M. (2009). Components of executive control with advantages for bilingual children in two cultures. Cognition, 112, 494-500.

Blanco-Elorrieta, E., \& Pylkkänen, L. (2016). Bilingual Language Control in Perception versus Action: MEG Reveals Comprehension Control Mechanisms in Anterior Cingulate Cortex and Domain-General Control of Production in Dorsolateral Prefrontal Cortex. The Journal of Neuroscience, $36(2), 290-301$.

Cattaneo, G., Calabria, M., Marne, P., Gironell, A., Abutalebi, J., \& Costa, A. (2015). The role of executive control in bilingual language production: A study with Parkinson's disease individuals. Neuropsychologia, 66, 99-110.

Calabria, M., Branzi, F. M., Marne, P., Hernández, M., \& Costa, A. (2015). Age-related effects over bilingual language control and executive control. Bilingualism: Language and Cognition, 18(01), 65-78.

Calabria, M., Hernández, M., Branzi, F. M., \& Costa, A. (2012). Qualitative differences between bilingual language control and executive control: evidence from task-switching. Frontiers in psychology, 2.

Christoffels, I. K., Firk, C., \& Schiller, N. O. (2007). Bilingual language control: An event-related brain potential study. Brain research, 1147, 192-208. 
Coderre, E. L., Smith, J. F., Van Heuven, W. J., \& Horwitz, B. (2015). The functional overlap of executive control and language processing in bilinguals. Bilingualism: Language and Cognition, 1-18)

Costa, A., \& Santesteban, M. (2004). Lexical access in bilingual speech production: Evidence from language switching in highly proficient bilinguals and L2 learners. Journal of Memory and Language, 50(4), 491-511.

Costa, A., Santesteban, M., \& Ivanova, I. (2006). How do highly proficient bilinguals control their lexicalization process? Inhibitory and language-specific selection mechanisms are both functional. Journal of Experimental Psychology: Learning, Memory, and Cognition, 32(5), 1057.

Costa, A., Hernández, M., \& Sebastián-Gallés, N. (2008). Bilingualism aids conflict resolution: Evidence from the ANT task. Cognition, 106(1), 59-86.

Costa, A., Hernández, M., Costa-Faidella, J., \& Sebastián-Gallés, N. (2009). On the bilingual advantage in conflict processing: Now you see it, now you don't. Cognition, 113(2), 135-149.

Cousineau, D., Brown, S., \& Heathcote, A. (2004). Fitting distributions using maximum likelihood: Methods and packages. Behavior Research Methods, Instruments, \& Computers, 36(4), 742-756.

De Baene, W., Duyck, W., Brass, M., \& Carreiras, M. (2015). Brain circuit for cognitive control is shared by task and language switching. Journal of Cognitive Neuroscience.

de Bruin, A., Bak, T. H., \& Della Sala, S. (2015). Examining the effects of active versus inactive bilingualism on executive control in a carefully matched non-immigrant sample. Journal of Memory and Language, 85, 15-26. 
de Bruin, A., Roelofs, A., Dijkstra, T., \& FitzPatrick, I. (2014). Domain-general inhibition areas of the brain are involved in language switching: FMRI evidence from trilingual speakers. NeuroImage, 90, 348-359.

Declerck, M., Thoma, A. M., Koch, I., \& Philipp, A. M. (2015). Highly Proficient Bilinguals Implement Inhibition: Evidence From n-2 Language Repetition Costs. Journal of Experimental Psychology: Learning, Memory, and Cognition, 41(6), 1911-1916.

Duñabeitia, J. A., Hernández, J. A., Antón, E., Macizo, P., Estévez, A., Fuentes, L. J. \& Carreiras, M. (2014). The inhibitory advantage in bilingual children revisited: Myth or reality? Experimental Psychology, 61 (3), 234- 251.

Festman, J., \& Münte, T. F. (2012). Cognitive control in Russian-German bilinguals. Front. Psychol, 3(115), 10-3389.

Festman, J., Rodriguez-Fornells, A., \& Münte, T. F. (2010). Individual differences in control of language interference in late bilinguals are mainly related to general executive abilities. Behavioral and Brain Functions, 6(1), 1.

Gollan, T. H., Sandoval, T., \& Salmon, D. P. (2011). Cross- language intrusion errors in aging bilinguals reveal the link between executive control and language selection. Psychological Science, 22(9), 1155-1164.

Goral, M., Campanelli, L., \& Spiro, A. (2015). Language dominance and inhibition abilities in bilingual older adults. Bilingualism: Language and Cognition, 18(01), 79-89.

Grange, J. A., \& Juvina, I. (2015). The effect of practice on $n-2$ repetition costs in set switching. Acta Psychologica, 154, 14-25.

Green, D. W. (1998). Mental control of the bilingual lexico-semantic system. Bilingualism: Language and cognition, 1(02), 67-81. 
Greenwood, P. M. (2000). The frontal aging hypothesis evaluated. Journal of the International Neuropsychological Society, 6, 705-726.

Guo, T., Ma, F., \& Liu, F. (2013). An ERP study of inhibition of non-target languages in trilingual word production. Brain and language, 127(1), 12-20.

Hartanto, A., \& Yang, H. (2016). Disparate bilingual experiences modulate taskswitching advantages: A diffusion-model analysis of the effects of interactional context on switch costs. Cognition, 150, 10-19.

Hernández, M., Martin, C. D., Barceló, F., \& Costa, A. (2013). Where is the bilingual advantage in task-switching?. Journal of Memory and Language, 69(3), 257276.

Jackson, G. M., Swainson, R., Cunnington, R., \& Jackson, S. R. (2001). ERP correlates of executive control during repeated language switching. Bilingualism: Language and Cognition, 4(02), 169-178.

Kiesel, A., Steinhauser, M., Wendt, M., Falkenstein, M., Jost, K., Philipp, A. M., \& Koch, I. (2010). Control and interference in task switching-A review. Psychological bulletin, 136(5), 849.

Koch, I., Gade, M., Schuch, S., \& Philipp, A. M. (2010). The role of inhibition in task switching: A review. Psychonomic Bulletin \& Review, 17(1), 1-14.

Kousaie, S., Sheppard, C., Lemieux, M., Monetta, L., \& Taler, V. (2014). Executive function and bilingualism in young and older adults. Frontiers in Behavioral Neuroscience, 8(250), 1-12. doi: 10.3389/fnbeh.2014.00250.

Lien, M. C., \& Ruthruff, E. (2008). Inhibition of task set: Converging evidence from task choice in the voluntary task-switching paradigm. Psychonomic Bulletin \& Review, 15(6), 1111-1116. 
Linck, J. A., Schwieter, J. W., \& Sunderman, G. (2012). Inhibitory control predicts language switching per-formance in trilingual speech production. Bilingualism: Language and Cognition, 15, 651-662.

Magezi, D. A., Khateb, A., Mouthon, M., Spierer, L., \& Annoni, J. M. (2012). Cognitive control of language production in bilinguals involves a partly independent process within the domain-general cognitive control network: Evidence from task-switching and electrical brain activity. Brain and Language, 122(1), 55-63.

Mayr, U. (2007). Inhibition of task sets. In D. S. Gorfein \& C. M. MacLeod (Eds.), Inhibition in cognition (pp. 27-44). Washington, DC: American Psychological Association.

Mayr, U., \& Keele, S. W. (2000). Changing internal constraints on action: the role of backward inhibition. Journal of Experimental Psychology: General, 129(1), 4.

Matzke, D., \& Wagenmakers, E. J. (2009). Psychological interpretation of the exGaussian and shifted Wald parameters: A diffusion model analysis. Psychonomic Bulletin \& Review, 16(5), 798-817.

McAuley, T., Yap, M., Christ, S. E., \& White, D. A. (2006). Revisiting inhibitory control across the life span: Insights from the ex-Gaussian distribution. Developmental neuropsychology, 29(3), 447-458.

Misra, M., Guo, T., Bobb, S. C., \& Kroll, J. F. (2012). When bilinguals choose a single word to speak: Electrophysiological evidence for inhibition of the native language. Journal of memory and language, 67(1), 224-237.

Moritz, S., Hübner, M., \& Kluwe, R. (2004). Task switching and backward inhibition in obsessive-compulsive disorder. Journal of Clinical and Experimental Neuropsychology, 26(5), 677-683. 
Paap, K. R., \& Greenberg, Z. I. (2013). There is no coherent evidence for a bilingual advantage in executive processing. Cognitive psychology, 66(2), 232-258.

Paap, K. R., \& Sawi, O. (2014). Bilingual advantages in executive functioning: problems in convergent validity, discriminant validity, and the identification of the theoretical constructs. Frontiers in psychology, 5(962), 2.

Paap, K. R., Johnson, H. A., \& Sawi, O. (2015). Should the search for bilingual advantages in executive functioning continue? Cortex, Doi:10.1016/j.cortex.2015.09.010.

Penner-Wilger, M., Leth-Steensen, C., \& LeFevre, J. A. (2002). Decomposing the problem-size effect: A comparison of response time distributions across cultures. Memory and Cognition, 30, 1160-1167.

Philipp, A. M., Gade, M., \& Koch, I. (2007). Inhibitory processes in language switching: Evidence from switching language-defined response sets. European Journal of Cognitive Psychology, 19(3), 395-416.

Philipp, A. M., \& Koch, I. (2006). Task inhibition and task repetition in task switching. European Journal of Cognitive Psychology, 18(4), 624-639.

Philipp, A. M., Jolicoeur, P., Falkenstein, M., \& Koch, I. (2007). Response selection and response execution in task switching: Evidence from a go-signal paradigm. Journal of Experimental Psychology: Learning, Memory, and Cognition, 33(6), 1062.

Prior, A. (2012). Too much of a good thing: Stronger bilingual inhibition leads to larger lag-2 task repetition costs. Cognition, 125(1), 1-12.

Prior, A., \& Gollan, T. H. (2011). Good language-switchers are good task-switchers: Evidence from Spanish-English and Mandarin-English bilinguals. Journal of the International Neuropsychological Society, 17(04), 682-691. 
Prior, A., \& Gollan, T. H. (2013). The elusive link between language control and executive control: A case of limited transfer. Journal of Cognitive Psychology, $25(5), 622-645$.

Prior, A., \& MacWhinney, B. (2010). A bilingual advantage in task switching. Bilingualism: Language and cognition, 13(02), 253-262.

Regev, S., \& Meiran, N. (2016). Cue response dissociates inhibitory processes: task identity information is related to backward inhibition but not to competitor rule suppression. Psychological Research, 1-14.

Rhodes, M. G. (2004). Age-related differences in performance on the Wisconsin card sorting test: A meta-analytic review. Psychology and Aging, 19, 482494.

Runnqvist, E., \& Costa, A. (2012). Is retrieval-induced forgetting behind the bilingual disadvantage in word production. Bilingualism: Language and Cognition, 15(2), 365-377.

Runnqvist, E., Strijkers, K., Alario, F., \& Costa, A. (2012). Cumulative semantic interference is blind to language: Implications for models of bilingual speech production. Journal of Memory and Language, 66(4), 850-869.

Scheil, J. (2016). Effects of absolute and relative practice on $n-2$ repetition costs. Acta psychologica, 164, 65-69.

Schmiedek, F., Oberauer, K., Wilhelm, O., Süß, H. M., \& Wittmann, W. W. (2007). Individual differences in parameters of reaction time distributions and their relations to working memory and intelligence. Journal of Experimental Psychology: General, 136(3), 414. 
Schuch, S., \& Koch, I. (2003). The role of response selection for inhibition of task sets in task shifting. Journal of Experimental Psychology: Human Perception and Performance, 29(1), 92.

Shao, Z., Roelofs, A., \& Meyer, A. S. (2012). Sources of individual differences in the speed of naming objects and actions: The contribution of executive control. The Quarterly Journal of Experimental Psychology, 65(10), 1927-1944.

Snodgrass, J. G., \& Vanderwart, M. (1980). A standardized set of 260 pictures: norms for name agreement, image agreement, familiarity, and visual complexity. Journal of experimental psychology: Human learning and memory, 6(2), 174.

Soveri, A., Rodriguez-Fornells, A., \& Laine, M. (2011). Is there a relationship between language switching and executive functions in bilingualism? Introducing a within-group analysis approach. Bilingualism and cognitive control, 138.

Spieler, D. H., Balota, D. A., \& Faust, M. E. (1996). Stroop performance in healthy younger and older adults and in individuals with dementia of the Alzheimer's type. Journal of Experimental Psychology: Human Perception and Performance, 22(2), 461.

Tao L., Marzecova A., Taft M., Asanowicz D., Wodniecka Z. (2011). The efficiency of attentional networks in early and late bilinguals: the role of age of acquisition Frontiers in Psychology. 2:123 10.3389/fpsyg.2011.00123.

Tse, C-S., \& Altarriba, J. (2014). The relationship between language proficiency and attentional control in Cantonese-English bilingual children: evidence from Simon, Simon switching, and working memory tasks. Frontiers in Psychology 5 . 
Verhaeghen, P., \& Cerella, J. (2002). Aging, executive control, and attention: A review of meta-analyses. Neuroscience and Biobehavioral Reviews, 26, 849857.

von Bastian, C. C., Souza, A. S., \& Gade, M. (2015). No Evidence for Bilingual Cognitive Advantages: A Test of Four Hypotheses. Journal of Experimental Psychology: General, 145 (2), 246-258.

Weissberger, G. H., Wierenga, C. E., Bondi, M. W., \& Gollan, T. H. (2012). Partially overlapping mechanisms of language and task control in young and older bilinguals. Psychology and Aging, 27(4), 959.

Weissberger, G. H., Gollan, T. H., Bondi, M. W., Clark, L. R., \& Wierenga, C. E. (2015). Language and task switching in the bilingual brain: Bilinguals are staying, not switching, experts. Neuropsychologia, 66, 193-203.

Whitmer, A. J., \& Banich, M. T. (2007). Inhibition versus switching deficits in different forms of rumination. Psychological science, 18(6), 546-553.

Wiseheart, M., Viswanathan, M., \& Bialystok, E. (2016). Flexibility in task switching by monolinguals and bilinguals. Bilingualism: Language and Cognition, 19(01), 141-146. 


\section{FIGURE CAPTIONS:}

Figure 1. Linguistic switching task. (a) Mean RTs relative to the three different types of sequence (CAA, CBA and ABA) and (b) Magnitude of the switch costs (n-1 shift cost and n-2 repetition cost). Errors bars refer to SE.

Figure 2. Non-linguistic switching task. (a) Mean RTs relative to the three different types of sequence (ABA, CBA and CAA) and (b) Magnitude of the switch costs (n-1 shift cost and n-2 repetition cost). Errors bars refer to SE.

Figure 3. Ex-Gaussian analysis. $\mu$ and $\tau$ values relative to the two costs (n-1 shift cost and n-2 repetition cost) in the (a) Linguistic switching task and in the (b) Non-linguistic switching task. Errors bars refer to SE.

Figure 4. Correlations between the $n-2$ repetition cost and the $n-1$ shift cost (RTs). (a) Linguistic switching task and (b) Non-linguistic switching task. 


\section{Table 1}

Languages' information and self-assessed proficiency in bilinguals.

\begin{tabular}{|l|c|c|c|}
\hline & L1 & L2 & L3 \\
\hline Age of Acquisition & $0.7(1.1)$ & $1.3(1.7)$ & $6.5(3.3)$ \\
\hline $\begin{array}{l}\text { Context of language } \\
\text { exposure }\end{array}$ & home, school & home, school & school \\
\hline Language proficiency & & & $5(0.9)$ \\
\hline Reading & $7(0.3)$ & $7(0.4)$ & $5(1.1)$ \\
\hline Writing & $6(0.7)$ & $6(0.8)$ & $4(1.1)$ \\
\hline Speaking & $7(0.6)$ & $6(0.7)$ & $5(1.1)$ \\
\hline Comprehension & $7(0.3)$ & $7(0.3)$ & \\
\hline & & & \\
\hline
\end{tabular}

Language proficiency scores were on a 7 point scale, in which 7 represents a very high level and 1 a very low level of proficiency. The self-assessed index is an average of participants' responses relative to each domain (reading, writing, speaking, and comprehension). In parentheses are reported SDs. 
Figure 1.

(a) Linguistic switching task

Mean RTs

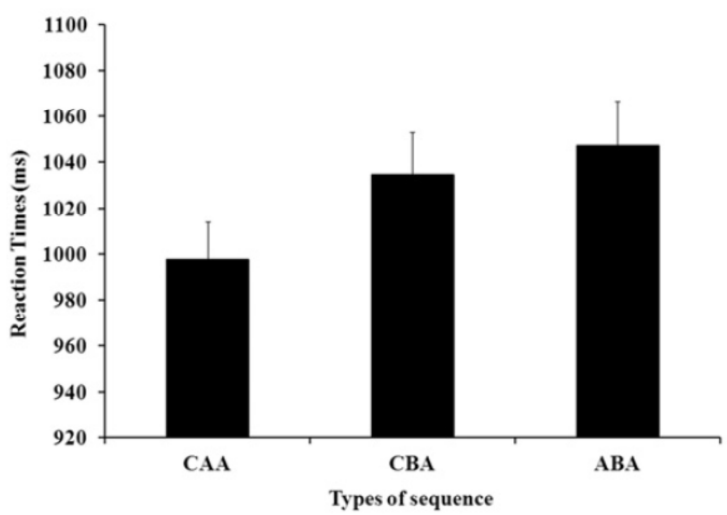

(b) Linguistic switching task Magnitude of Switch Costs

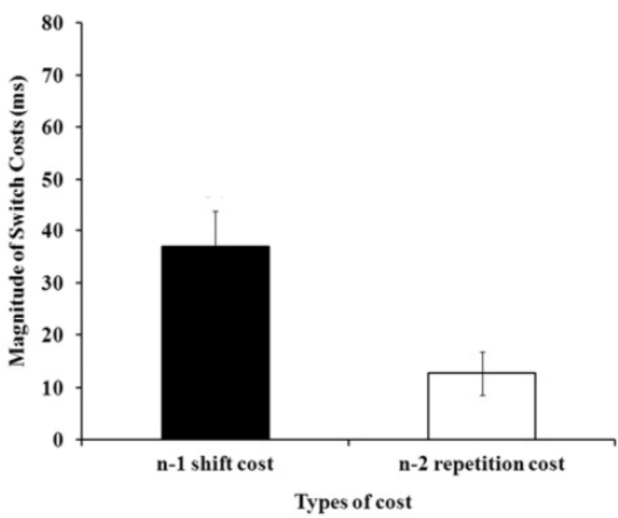

Figure 2.

(a) Non-linguistic switching task Mean RTs

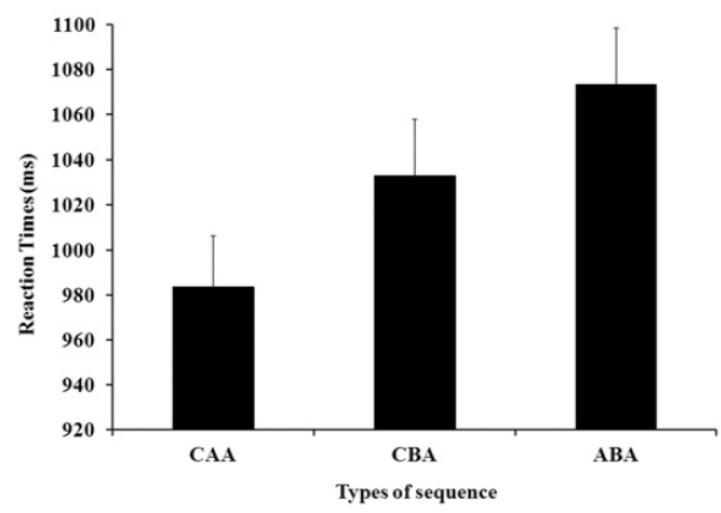

(b) Non-linguistic switching task Magnitude of Switch Costs

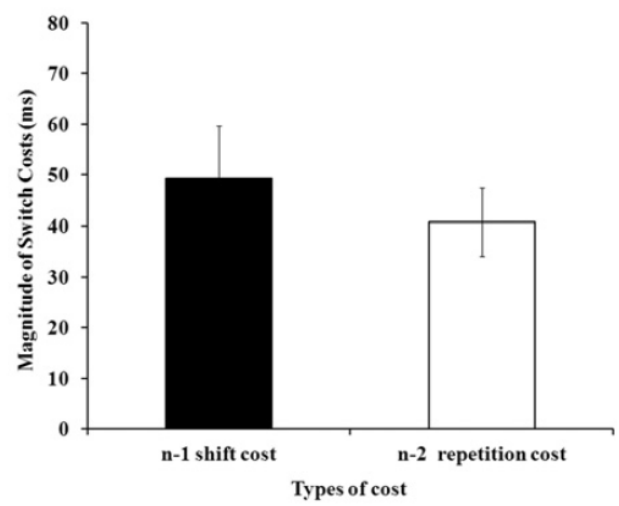


Figure 3.

(a) Linguistic switching task: $\mu$ and $\tau$ values

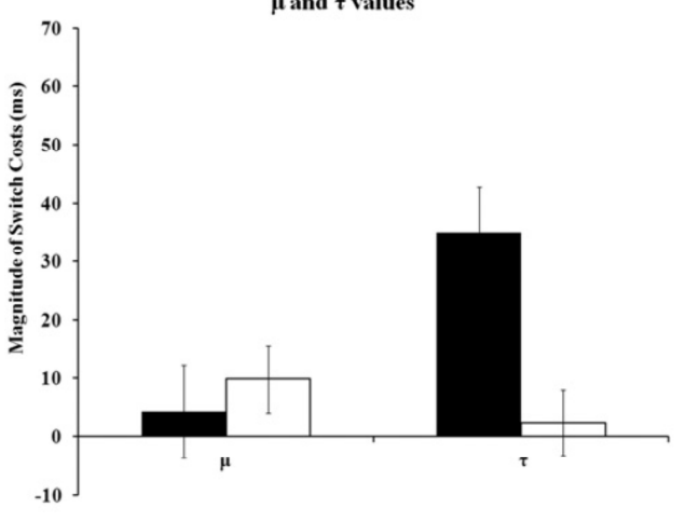

m-1 shift cost (b) Non-linguistic switching task:

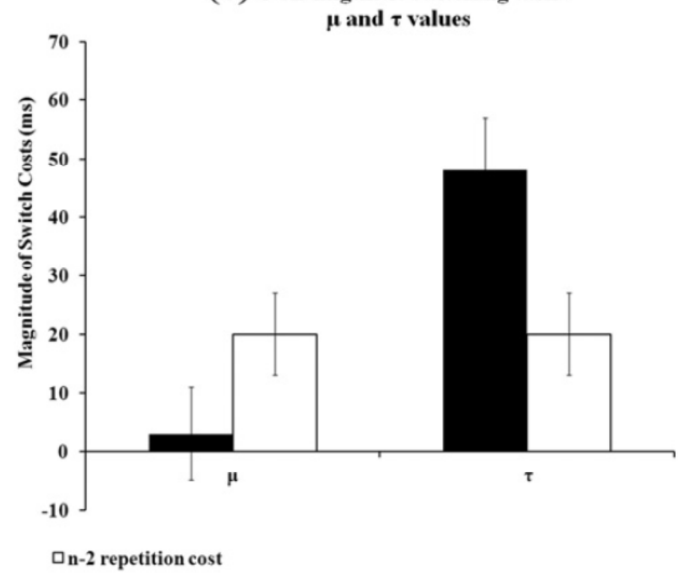

\section{Figure 4.}

(a) Linguistic switching task:

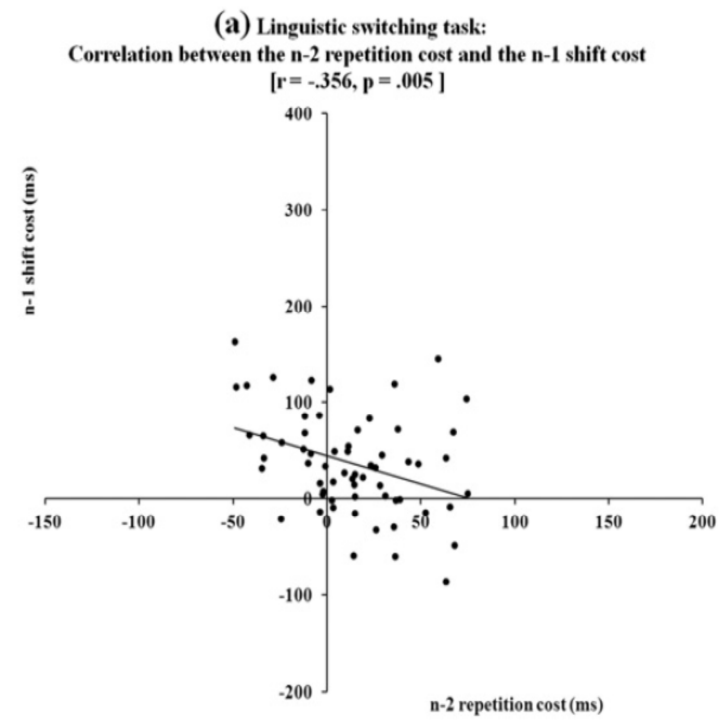

(b) Non-linguistic switching task: Correlation between the $n-2$ repetition cost and the $n-1$ shift cost

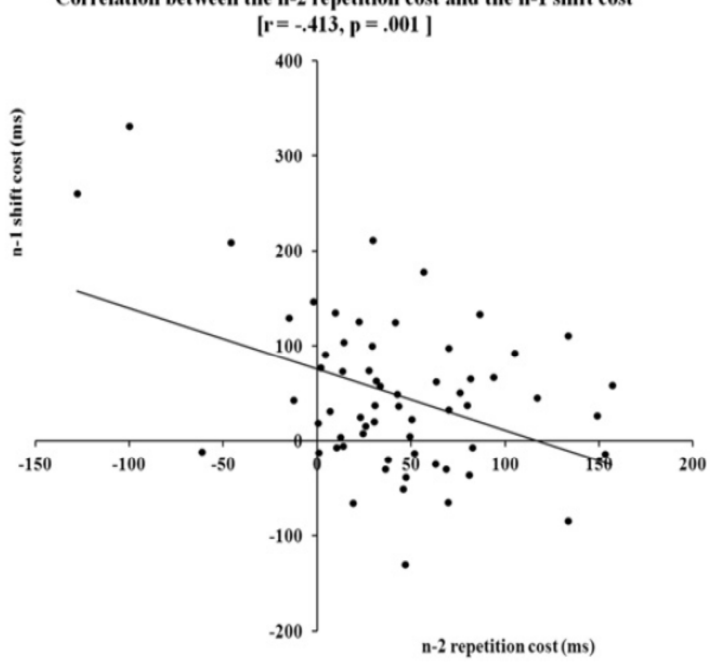

